\title{
Serotype-specific Monoclonal Antibodies against the H12 Flagellar Antigen of Escherichia coli
}

\author{
By CHRISTOPHER WHITFIELD, ${ }^{1 *}$ STEPHEN G. WALKER, ${ }^{1}$ \\ CHRISTOPHER F. ATKINSON, ${ }^{1}$ JOSEPH S. LAM, ${ }^{1}$ \\ LESLIE A. MACDONALD, ${ }^{1}$ TERRY J. BEVERIDGE, ${ }^{1}$ IDA ØRSKOV ${ }^{2}$ \\ AND FRITS ØRSKOV ${ }^{2}$ \\ ${ }^{1}$ Department of Microbiology, College of Biological Sciences, University of Guelph, Guelph, \\ Ontario NIG 2Wl, Canada \\ ${ }^{2}$ Collaborative Centre for Reference and Research on Escherichia coli (World Health \\ Organization), Statens Seruminstitut, DK-2300 Copenhagen, Denmark
}

(Received 25 September 1987; revised 17 February 1988)

\begin{abstract}
The flagellar filaments of morphotype $\mathrm{E}$ isolates of Escherichia coli characteristically possess an apparent helically arranged sheath structure, surrounding the central core of the filament. Reexamination of the type strains of $\mathrm{H}$-serotypes belonging to morphotype $\mathrm{E}$ showed that all but serotype $\mathrm{H} 34$ possessed the expected morphology. Heterogeneity was observed in both the diameter of filaments from individual morphotype $\mathrm{E}$ strains and in the $M_{\mathrm{r}}$ of individual flagellins. There was no apparent correlation between these two features. Monoclonal antibodies (MAbs) of the IgM class were raised against serotype $\mathrm{H} 12$ flagella. In Western immunoblotting and agglutination tests, the MAbs recognized the $\mathrm{H} 12$ antigen of six isolates with different $\mathrm{O}: \mathrm{K}$ antigen combinations. The MAbs were $\mathrm{H}$-serotype-specific, with no significant reaction with the $\mathrm{H}$-antigens of other morphotype $\mathrm{E}$ strains. The location of the serotype-specific $\mathrm{H} 12$ epitope(s) was studied by immunolabelling with colloidal gold markers. The epitope was surface-exposed and appeared to be helically arranged on the flagellar filament. The pattern of colloidal gold labelling was consistent with the possibility that the H12 serotypespecific epitope resides in the apparent sheath structure.
\end{abstract}

\section{INTRODUCTION}

Flagella are organelles of locomotion of bacterial cells. The complete flagellar structure is comprised of three regions, the basal body, the hook, and the flagellar filament (MacNab, 1987). The filament is assembled from many copies of a single protein subunit, flagellin, arranged in approximately eleven longitudinal rows of subunits. The overall effect is a thread-like structure of uniform thickness (approximately $20 \mathrm{~nm}$ ) and a length in the range of 5-10 $\mu \mathrm{m}$ (MacNab, 1987).

The flagellar filament possesses exposed antigenic determinants ( $\mathrm{H}$-antigens), which allow serological distinction between different $\mathrm{H}$-serotypes. There are 53 recognized $\mathrm{H}$-serotypes of Escherichia coli (Ørskov \& Ørskov, 1984). Of these, $45 \mathrm{H}$-serotypes can be grouped into six morphological groups (designated morphotypes $\mathrm{A}-\mathrm{F}$ ), based on the appearance of the flagella under the electron microscope (Lawn et al., 1977). Flagella belonging to morphotypes $\mathrm{E}$ and $\mathrm{F}$ are perhaps the most complex morphologically, due to the presence of an apparent helical sheath structure surrounding the central core of the filament. Eight $\mathrm{H}$-serotypes have flagella with morphotype $\mathrm{E}$ filaments and a further $15 \mathrm{H}$-serotypes exhibit morphotype $\mathrm{F}$ (Lawn et al., 1977).

Abbreviation: MAb, monoclonal antibody.

0001-4431 (C) 1988 SGM 
No separate sheath protein has been identified and in morphotype $\mathrm{E}$ and $\mathrm{F}$ filaments available evidence suggests that the central core and the apparent sheath are both formed from the same flagellin subunits (Lawn, 1977). The $M_{\mathrm{r}}$ values of the flagellin subunits in $E$. coli vary from 37000-69000 and correlate with the diameters of the filaments when compared across the different morphotypes (Lawn, 1977). Serological cross-reactions among $\mathrm{H}$-antigens have been noted and these generally occur between serotypes within the same morphotype (Lawn et al., 1977).

We are interested in the molecular basis for $\mathrm{H}$-antigen diversity in E. coli, particularly in strains possessing complex morphotype $E$ and $F$ flagella. The objective of the studies reported here was to investigate the role of the apparent sheath structure in determination of $\mathrm{H}$-antigen specificity in serotype $\mathrm{H} 12$ flagellar filaments representative of morphotype E.

\section{METHODS}

Bacterial strains and growth media. The bacterial strains used in this study are listed in Table 1. All strains were obtained from the collection of the Collaborative Centre for Reference and Research on Escherichia coli (WHO), Copenhagen, Denmark. Cultures were maintained as frozen stocks and grown in Luria broth (LB: Bacto-tryptone, $10 \mathrm{~g} \mathrm{l}^{-1}$; Bacto yeast extract, $5 \mathrm{~g} \mathrm{l}^{-1} ; \mathrm{NaCl}, 5 \mathrm{~g} \mathrm{l}^{-1}$ ) at $37^{\circ} \mathrm{C}$. All strains were passed through semi-solid motility agar (LB containing $0.2 \%$ agar) prior to use, to ensure that motile forms were selected.

Isolation of flagella. For electron microscopy and immunogold labelling, flagella with intact basal bodies were isolated using the method described by Johnson et al. (1979). Briefly, this procedure involves Triton X-100 lysis of lysozyme and EDTA-treated mid-exponential phase cells. Intact flagella comprising basal body, hook and filament are obtained from DNAase-treated cell lysates, using differential centrifugation.

Flagellin preparations. For analysis of flagellins from a number of bacterial strains, a rapid technique was adopted. Cultures were grown on aluminium trays $(40 \times 25 \mathrm{~cm})$ containing $500 \mathrm{ml}$ of solid medium. The bacterial growth was scraped off and resuspended in $150 \mathrm{ml} 0 \cdot 1 \mathrm{M}-\mathrm{HEPES} / \mathrm{NaOH}$ buffer, $\mathrm{pH} \mathrm{6.8}$. All subsequent steps were done at $4{ }^{\circ} \mathrm{C}$. Flagella were removed from the cells by treatment for 1-2 $\mathrm{min}$ in a Waring blender. The cells and large membrane fragments were removed from suspension by successive centrifugation steps at $7500 \mathrm{~g}$ for $15 \mathrm{~min}$ and $9500 \mathrm{~g}$ for $15 \mathrm{~min}$, respectively. Flagella and remaining membrane contaminants were harvested from the cellfree supernatant fraction by ultracentrifugation at $200000 \mathrm{~g}$ for $1 \mathrm{~h}$. The pellet was resuspended in approximately $0.5 \mathrm{ml} 0.1 \mathrm{M}$-HEPES/NaOH, $\mathrm{pH} \mathrm{6.8}$. Flagellar filaments were converted to flagellin monomers by heating at $90^{\circ} \mathrm{C}$ for $10 \mathrm{~min}$. Purified flagellin was then obtained following centrifugation in a Beckman Airfuge at $120000 \mathrm{~g}$ for $20^{\circ}$ min, which removed membrane contaminants as a pellet and yielded highly purified flagellin in the supernatant fraction. The degree of purity increased when the supernatant was subjected to a second heating and Airfuge centrifugation cycle. The preparations were essentially free of contaminants as judged by SDS-PAGE and

Table 1. E. coli strains used in this study

\begin{tabular}{|c|c|c|c|c|}
\hline Strain & Serotype & $\begin{array}{c}\text { Reference strain } \\
\text { H-serotype }\end{array}$ & Flagellin $M_{\mathrm{r}}^{*}$ & $\begin{array}{c}\text { Filament } \\
\text { diameter }(n m) \dagger\end{array}$ \\
\hline Su 1242 & $\mathrm{O} 2: \mathrm{K} 2: \mathrm{H} 1$ & H1 & 67000 & 21 \\
\hline U5-41 & $\mathrm{O} 1: \mathbf{K} 1: \mathbf{H} 7$ & H7 & 67000 & 19 \\
\hline Bi $316-42$ & O9:K9:H12 & H12 & 66000 & 21 \\
\hline $\mathrm{K} 42$ & $\mathrm{O} 45: \mathrm{K} 1: \mathrm{H} 23$ & $\mathrm{H} 23$ & 67000 & 19 \\
\hline BP 12665 & O86:K ?:H34 & H34t & 67000 & 23 \\
\hline $4106-54$ & $\mathrm{O} 52: \mathrm{K} ?: \mathrm{H} 45$ & H45 & 61000 & 19 \\
\hline $2147-59$ & O6:K13:H49 & H49 & 60000 & 19 \\
\hline C218-70 & O8:K50:H51 & H51 & 65000 & 17 \\
\hline E69 & $\mathrm{O} 9: \mathrm{K} 30: \mathrm{H} 12$ & & 66000 & 21 \\
\hline $\mathrm{E} 47 \mathrm{a}$ & $\mathrm{O} 25: \mathrm{K} 19: \mathrm{H} 12$ & & 66000 & 21 \\
\hline U12-41 & $\mathrm{O} 49: \mathrm{K} ?: \mathrm{H} 12$ & & 66000 & 21 \\
\hline $\mathrm{P} 10 \mathrm{a}$ & $\mathrm{O} 71: \mathrm{K} ?: \mathrm{H} 12$ & & 66000 & 21 \\
\hline $\mathrm{H} 40$ & $\mathrm{O} 87: \mathrm{K} ?: \mathrm{H} 12$ & & 66000 & 21 \\
\hline
\end{tabular}

\footnotetext{
* Mean of three or more determinations; in each case, the determinations varied \pm 1000 .

$\dagger$ Mean of measurements from 10 or more separate filaments; in each case, the determination varied $\pm 0 \cdot 1 \mathrm{~nm}$. $\ddagger$ Morphology differs from the other strains listed (see text).

NB Lawn (1977) has reported analysis of flagella from strains Su 1242 (diameter $24 \mathrm{~nm} ; M_{\mathrm{r}} 60000$ ), U5-41 $(23 \mathrm{~nm} ; 61000)$ and $\mathrm{Bi} 316-42(22 \mathrm{~nm} ; 62000)$.
} 
Coomassie brilliant blue staining. Protein contents were determined by the Lowry method. The yield of flagellin depended on the strain examined. Routinely $3-8 \mathrm{mg}$ of purified flagellin was obtained from a single tray of culture.

Monoclonal antibody $(M A b)$ production. Two female Balb/c mice (8-10 weeks old) were each immunized subcutaneously on day 1 with $100 \mu \mathrm{g}$ of purified flagella, suspended in Freund's incomplete adjuvant. Strain E69 was the source of the antigen. On day $10,50 \mu \mathrm{g}$ of flagella was administered intravenously. Fusions were done on day 13. Spleen cells from immunized mice were fused with NS-1 murine myeloma cells following the procedure detailed by Lam et al. $(1987 b)$. Positive hybridomas were identified by enzyme-linked immunosorbent assay (ELISA) with flagella as antigen. Wells were coated using flagella $\left(5 \mu \mathrm{g} \mathrm{ml}^{-1}\right)$ in carbonate buffer (Voller et al., 1976). The blocking agent was $3 \%$ BSA (Sigma) in phosphate-buffered saline (PBS) (Voller et al., 1976). Goat antimouse $\mathrm{F}(\mathrm{ab})_{2}$ conjugated to alkaline phosphatase (Helix Biotech) was used with $p$-nitrophenyl phosphate substrate in the detection system. Positive hybridomas were cloned by limiting dilution. All further experiments were done using hybridoma supernatants. MAb class and subclass were determined using the mouse typer kit from Bio-Rad.

$S D S-P A G E$. Flagellin preparations were analysed by SDS-PAGE according to the method of Laemmli (1970), using resolving gels containing $10 \%(\mathrm{w} / \mathrm{v})$ acrylamide and $4 \%$ stacking gels. Samples were dissociated in Laemmli sample buffer by heating at $95-100^{\circ} \mathrm{C}$ for $10 \mathrm{~min}$ immediately before electrophoresis. Protein $M_{\mathrm{r}}$ standards were obtained from Bio-Rad. Approximately $1 \mu \mathrm{g}$ of purified flagellin was loaded per lane.

Western immunoblotting. Flagellin samples were transferred from SDS-PAGE gels to nitrocellulose using modifications (Burnette, 1981) of the method originally described by Towbin et al. (1979). Immunoblots were developed using peroxidase-coupled, goat anti-mouse IgG and IgM (Helix Biotech). The colour reagent was 4chloro-1-naphthol (Sigma).

Slide agglutination reactions. Slide agglutination reactions were performed using overnight cultures. Bacterial growth was scraped from a plate using a sterile toothpick and resuspended in a $0.01 \mathrm{ml}$ drop of saline on a glass microscope slide. Hybridoma supernatant $(0.001 \mathrm{ml})$ was added and mixed with the cell suspension. Clumping of bacterial cells, due to a positive reaction, was evident in less than $20 \mathrm{~s}$.

Preparation of colloidal gold markers. Colloidal gold markers, $12.5 \mathrm{~nm}$ in diameter, were coupled to diaminoethane-derivatized dextran using the methods described in detail by Lam et al. (1987a). The marker was then coupled to Protein A (Boehringer Mannheim) as described by Hicks \& Molday (1986).

Colloidal gold labelling. Copper grids (100 mesh) were coated with carbon and formvar and treated with $0 \cdot 1 \%$ peptone as a wetting agent. Flagella samples contained in a drop of PBS were added to the grid and allowed to dry. The grids were blocked using 3\%(w/v) bovine serum albumin in PBS for $15 \mathrm{~min}$ and the grid was then washed by inverting on successive drops of PBS. MAb H12-1 $(0.05 \mathrm{ml})$ was added to the grid which was then incubated in a moist chamber at room temperature for $30 \mathrm{~min}$. The grid was then washed exhaustively over drops of PBS containing $0.5 \%$ BSA. A second antibody $(0.05 \mathrm{ml}$ goat anti-mouse IgM; Cappel Worthington Biochemicals) was then added and allowed to react at room temperature for $30 \mathrm{~min}$. The grid was again washed extensively before addition of the Protein-A-coupled gold marker ( $0.08 \mathrm{ml})$. After incubation at room temperature for a further 30 min, the grid was washed and negatively stained. Controls included samples where either the first or second antibody were omitted.

Negative staining and electron microscopy. Samples were negatively stained by inverting the grids over a drop of $0.5 \%$ ammonium molybdate, $\mathrm{pH} 7.0$, for $5 \mathrm{~s}$. Use of a negative stain at neutral $\mathrm{pH}$ avoided problems with dissociation of flagellar filaments seen with uranyl acetate staining (Lawn et al., 1977). Excess stain was removed using filter paper. The grids were examined in a Philips 300 transmission electron microscope operating at $60 \mathrm{kV}$.

\section{RESULTS}

\section{Production and specificity of anti-H12 MAbs}

Hybridomas from fusion experiments were screened initially by ELISA with intact $\mathrm{H} 12$ flagella as antigen. Intact flagella were used in an attempt to ensure that only MAbs recognizing surface-exposed epitopes on the flagellar filaments were selected for further study. More than 100 clones were obtained from each of two fusion experiments and three MAbs (designated H12-1, H12-2 and H12-3) were studied further. These three hybridomas were selected since they yielded tissue culture supernatants with high titres of secreted MAb $\left(A_{405}\right.$ greater than $0 \cdot 1$ at a $10^{-8}$ dilution in ELISA); these supernatants were the source of MAb in all subsequent experiments. All the MAbs analysed belonged to class IgM and their reactivities were, on subsequent analysis, indistinguishable. Only the results obtained for MAb H12-1 are reported below.

The serotype specificity of the MAbs was determined by slide agglutination reactions and by Western immunoblotting. All three MAbs gave positive results in slide agglutination tests with the homologous strain (E. coli E69). When tested against the H12 type strain (Bi 316-42) and 
(a)

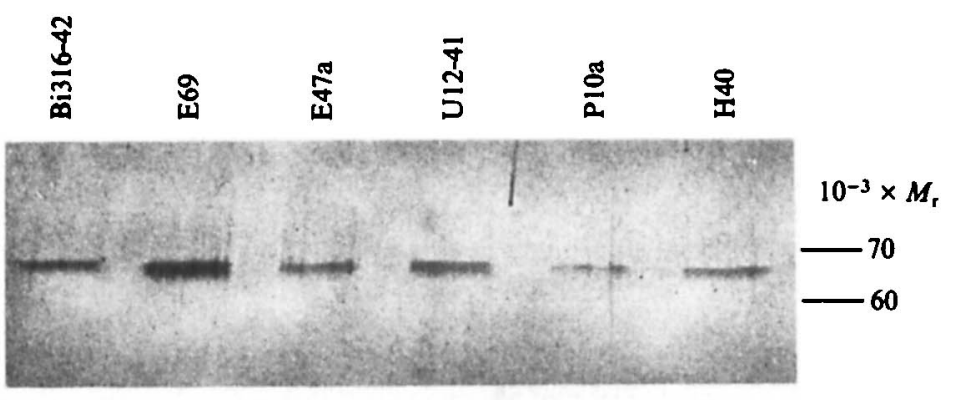

(b)

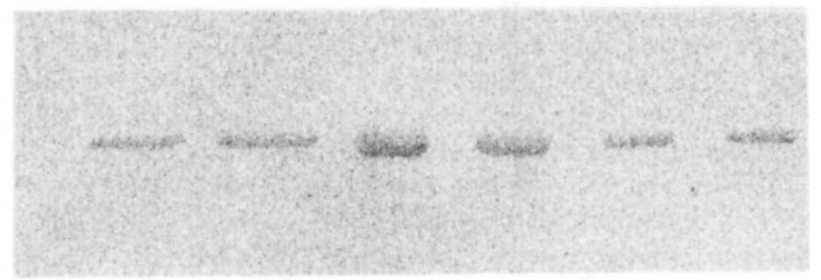

(c)

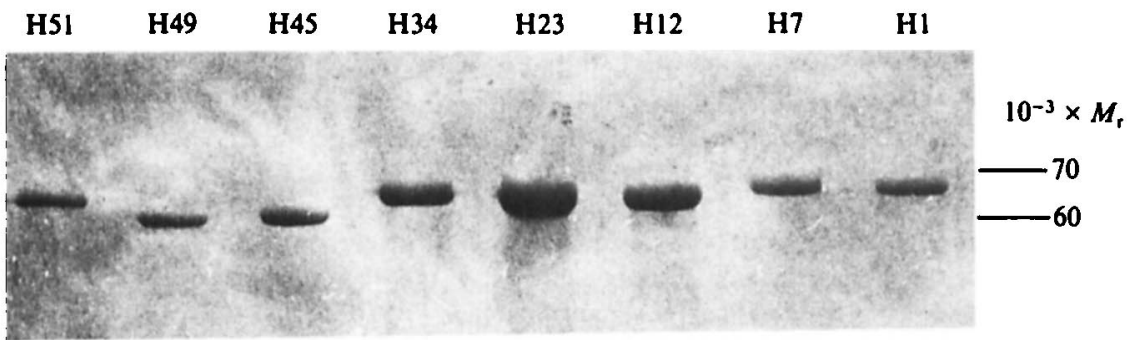

(d)

Fig. 1. SDS-PAGE and Western immunoblotting of flagellins from morphotype E strains of $E$. coli. Flagellins prepared from $\mathrm{H} 12$ isolates are shown in $(a)$. Strain numbers are indicated above the appropriate lanes. In $(b)$, an immunoblot from the same gel was probed using MAb H12-1. In $(c)$, the flagellins from each of the morphotype E H-serotype reference strains were analysed by SDS-PAGE. The lanes are labelled according to $\mathrm{H}$-serotype. The immunoblot from the same gel, probed using MAb H12-1, is shown in (d). Flagellin samples were prepared and analysed as described in Methods. Each lane contained approximately $1 \mu \mathrm{g}$ protein and only the relevant portion of the gel is shown. For details of strains and flagellin $M_{\mathrm{r}}$, see Table 1 .

against five other H12 serotype strains (E69, E47a, U12-41, P10a and H40), the MAbs again gave positive slide agglutination results. None of the MAbs agglutinated other $\mathrm{H}$-serotype strains belonging to morphotype E. Western immunoblotting was also used to evaluate the specificity of the MAbs, in order to check for weak cross-reactions which might not be detectable by agglutination. MAb H12-1 gave a positive reaction against the flagellins of six different H12-serotype strains (Fig. $1 a, b$ ). Only the flagellin band was recognized by this MAb regardless of whether purified flagellin, crude flagellar preparations or membrane fractions were probed (results not shown). When H12-1 MAb was used to probe the flagellins from the eight $\mathrm{H}$ serotype reference strains belonging to morphotype $\mathrm{E}$ (Fig. $1 c, d$ ), only the $\mathrm{H} 12$ antigen was 


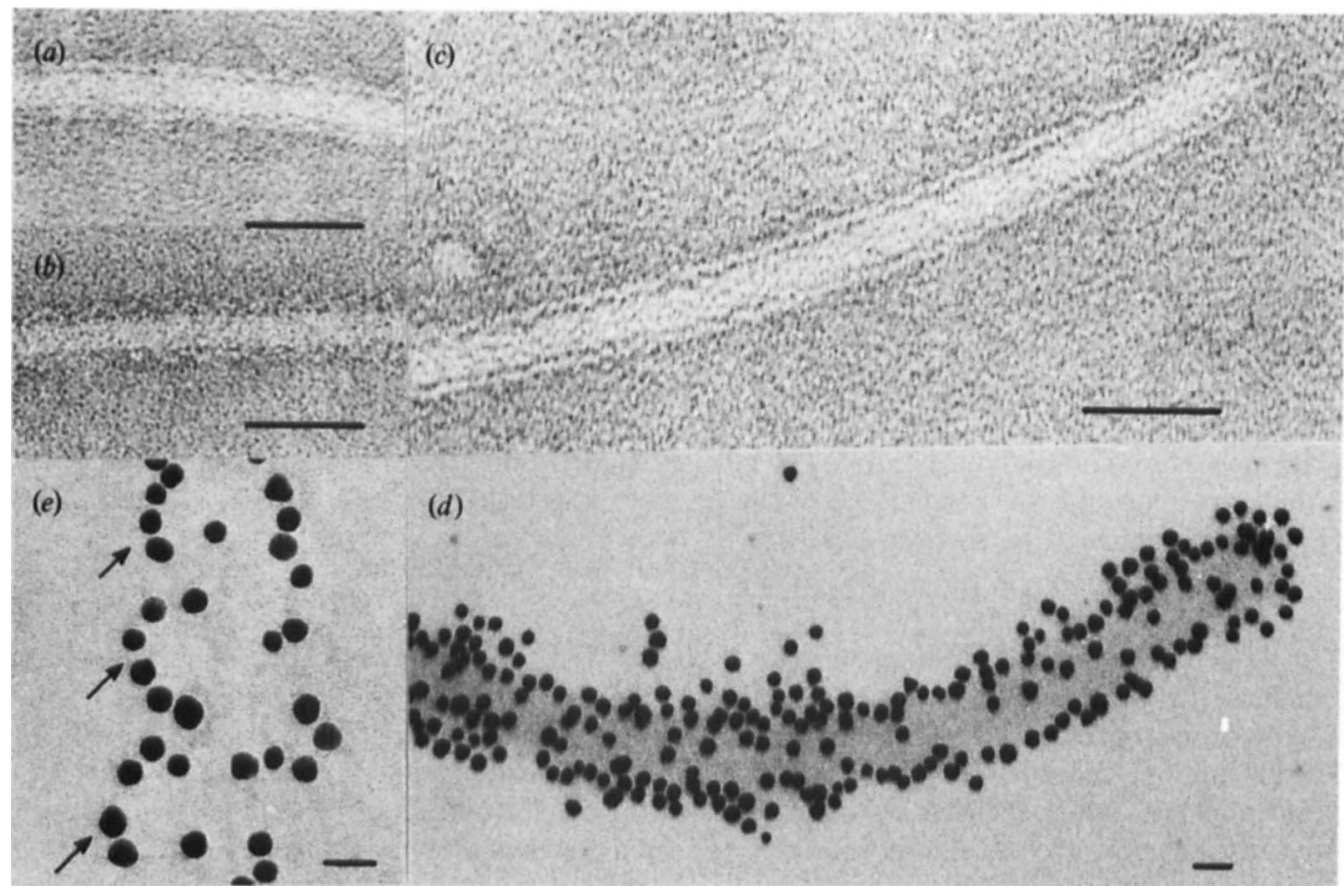

Fig. 2. Morphology of morphotype E flagellar filaments and immunolabelling of the serotype-H12specific antigenic epitope. $(a, c)$ Negatively stained serotype $\mathrm{H} 12$ filaments, typical of morphotype $\mathrm{E}$. $(b)$ Filaments from serotype $\mathrm{H} 34$. $(d, e)$ Immunogold labelling of serotype $\mathrm{H} 12$ flagellar filaments. In $(e)$, the edges of successive periodic loops of the apparent sheath, labelled with gold, are indicated by arrows. Bars, $40 \mathrm{~nm}$.

clearly detected. A very slight reaction was consistently seen against the $\mathrm{H} 45$ flagellin. This reaction was so weak that even with overloading of antigen, the reaction was not visible in photographs of the resulting immunoblots. An $\mathrm{H}$-serotype strain representing each of the other morphotypes was also tested (results not presented) and no reaction was observed with MAb H12-1 in either agglutination or Western immunoblot tests. The strains tested were $E$. coli U9-41 (serotype H4, morphotype A), U4-41 (serotype H5, morphotype B), P4 (serotype H48, morphotype C), Ulla-44 (serotype H21, morphotype D) and 5306-56 (serotype H46, morphotype F).

\section{Morphology of H12 and H34 flagellar filaments}

Although the flagellar filaments of morphotype $\mathrm{E}$ have been reported to be morphologically identical (Lawn et al., 1977), analysis of the different flagellins showed some heterogeneity in $M_{\mathrm{r}}$ (Fig. 1 $c$; ; Table 1). In order to ensure that the filaments analysed were indeed morphotype E, samples were examined by electron microscopy: flagella from seven of the eight morphotype $E$ strains possessed similar morphology. In typical morphotype $\mathrm{E}$ strains, exemplified by serotype H12 (Fig. $2 a, c$ ), the apparent sheath is readily visible as a structure which appears to completely envelope the central core of the filament. The individual helically arranged loops of the apparent sheath are not readily resolved in typical micrographs. Diagrammatic representations have been presented elsewhere by Lawn et al. (1977). One strain, the type strain for H34, produced flagellar filaments with a slightly different morphology (Fig. $2 b$ ). In this isolate, the sheath structure comprised larger and more readily visible loops at the edge of the filament and the periodicity of the loops was also different to that of the other morphotype E strains. No filaments devoid of the sheath structure were observed in any of the samples analysed and the morphology for a given isolate was independent of growth conditions.

Electron microscopy also demonstrated that the individual filaments from the different 
morphotype $\mathrm{E} \mathrm{H}$-serotypes were heterogeneous in terms of their diameters (Table 1). No correlation was found between filament diameter and flagellin $M_{\mathrm{r}}$.

\section{Antigenic epitopes of $\mathrm{H} 12$ flagellar flaments}

In an attempt to localize the antigenic epitope on the H12 flagellar filaments, MAb H12-1 was used as a probe coupled with immunogold labelling. As shown in Fig. $2(d)$, the gold marker specifically labelled the surface of the filaments. It was necessary to reduce the staining of the filament in gold-labelling experiments, in order to make the probe clearly visible. As a result, details of the surface structure of the filament could not be resolved in gold-labelled specimens. Interestingly, the gold marker also labelled the distal tip of the flagellar filament or filament fragments (Fig. 2d). Close examination of the gold-labelled filaments (Fig. 2e) showed many areas in which the gold marker followed a helical path around the filament and the periodicity of the looped structure at the surface of the filament was clearly visible. In Fig. 2(e), successive loops are indicated by arrows.

\section{DISCUSSION}

Flagellar filaments from morphotype E strains of $E$. coli are characterized by an apparent helical sheath structure with a $10 \mathrm{~nm}$ edge repeat (Lawn et al., 1977). In this report we have examined representative $\mathrm{H}$-serotype strains possessing morphotype $\mathrm{E}$ flagella. On reexamination, the filaments from the $\mathrm{H} 34$ type strain were found to be slightly different in morphology, when compared to other morphotype E representatives. The H34 filaments resembled more closely the morphology expected for morphotype F (Lawn et al., 1977). The reason for this inconsistency remains unclear as the strain used in this and previous (Lawn et al., 1977) reports is the same. In the original report (Lawn et al., 1977), flagella were fixed in formalin prior to staining in order to minimize the dissociation of acid-labile filaments in acidic uranyl acetate. To avoid these problems, we have used ammonium molybdate at neutral $\mathrm{pH}$ for negative staining. Differences in H34 flagellar filament morphology between these studies may therefore result from altered staining procedures and the absence of formalin fixation. These same differences in technique may explain differences in filament diameter in several morphotype E flagella (see Table 1 and footnote) when compared to the values reported by Lawn (1977).

Examination of filament diameters and flagellin $M_{\mathrm{r}}$ values demonstrated heterogeneity among the various representative strains of morphotype $E$. No correlation was evident between these two measurements, even though it has been shown previously that there is a correlation between filament diameter and flagellin $M_{\mathrm{r}}$ when different morphotypes are considered (Lawn, 1977). The $M_{\mathrm{r}}$ values of the flagellins from different morphotypes range from 37000 in morphotype A to 69000 in the unique morphotype represented by flagella of serotype H36. In comparison, the range of flagellin $M_{\mathrm{r}}$ values within morphotype $\mathrm{E}$ is from 60000 to 67000 . It is possible, although unlikely, that inaccuracies in measurement of filament diameters, coupled with the limited heterogeneity in flagellin $M_{\mathrm{r}}$ obscures any relationship within the group. Indeed, Lawn (1977) also examined three representative strains of morphotype $E$ and again within those particular samples, the correlation was not evident. It therefore appears from these results that the diameters of flagellar filaments in individual morphotype $E$ strains do not depend simply on the $M_{\mathrm{r}}$ of the component flagellin subunits. Differences in $M_{\mathrm{r}}$ described in this communication when compared to the previous report may be ascribed to the use of slab gels rather than tube gels and calibration with more markers of known $M_{\mathrm{r}}$.

Cross-reactions between different $\mathrm{H}$-serotypes have been reported and these often occur between serotypes with morphologically identical flagellar filaments (Lawn et al., 1977). A common cross-reaction is between serotypes H1 and H12 (Ewing, 1986; Ørskov \& Ørskov, 1984). The MAbs reported here reacted only with $\mathrm{H} 12$ and not with $\mathrm{H} 1$ flagellin. Although a slight reaction was observed with $\mathrm{H} 45$ flagellin in Western blots, this reaction was weak and insufficient to give a cross-reaction in agglutination experiments. Thus, the MAbs demonstrated precise specificity and may prove to be a useful alternative to absorbed polyclonal sera in $\mathrm{H}$ serotyping. 
The epitope recognized by the H12 serotype-specific MAb appears to reside in the apparent sheath structure of the filament, with the gold marker labelling an epitope with the helical and periodic features expected of the sheath. The gold marker was exclusively associated with the filament and no labelling of the basal body was seen in any specimen (results not shown). In some specimens (Fig. $2 c, d$ ), free distal tips of filaments were evident. It is not clear whether these tips represent the filament end occurring in vivo, or a product of fragmentation during preparation. Interestingly, the gold probe gave extensive labelling of the tips of filaments, suggesting that even at the tips the central filament core may be covered by H12-specific epitope. In terms of antigenic diversity and strategies to escape host immune response, such an arrangement would make sense. It is likely that the central core of the filament contains conserved regions of the flagellin, as this region must fulfil the same function (i.e. assembly into a functional filament) in all $\mathrm{H}$-serotypes. Accordingly, a high degree of homology has been reported between both the amino- and carboxy-termini of flagellins from various bacterial sources (Kuwajima et al., 1986). It would be a selective advantage to the bacterium to keep these conserved regions of the filament in a form which is inaccessible to antibody. In Salmonella, the antigenic (and surface exposed) region may reside towards the centre of the flagellin amino acid sequence (MacNab, 1987).

With the isolation of H12-serotype-specific MAbs and the indication that the corresponding epitope resides in the apparent sheath structure, it may now be possible to begin to define the antigenic domains within flagellin subunits and the organization of these epitopes when the subunits are assembled into flagellar filaments. Such studies should lead to a better understanding of the molecular basis for antigenic diversity in the flagellar antigens of $E$. coli.

The authors gratefully acknowledge the technical assistance of Sheila Wilmot and Gary Schoenhals. Colloidal gold markers were kindly provided by Michele Lam. Electron microscopy was performed at the NSERC Regional STEM facility at the University of Guelph. This work was supported by research operating grants from NSERC to C.W. and J.S.L.

\section{REFERENCES}

BURNETTE, W. N. (1981). "Western blotting": electrophoretic transfer of proteins from sodium dodecyl sulfate-polyacrylamide gels to unmodified nitrocellulose and radiographic detection with antibody and radioiodinated protein A. Analytical Biochemistry 112, 195-203.

EwING, W. H. (1986). The Genus Escherichia. In Edwards and Ewing's Identification of Enterobacteriaceae, 4th edn, pp. 93-136. Edited by W. H. Ewing. Amsterdam: Elsevier.

Hicks, D. \& Molday, R. S. (1986). Differential immunogold-dextran labeling of bovine and frog rod and cone cells using monoclonal antibodies against bovine rhodopsin. Experimental Eye Research 42, 55-71.

Johnson, R. C., Walsh, M. P., Ely, B. \& Shapiro, L. (1979). Flagellar hook and basal complex of Caulobacter crescentus. Journal of Bacteriology 138, 984989.

Kuwajima, G., Asaka, J.-I., Fujiwara, T., Fujiwara, T., Node, K. \& Kondo, E. (1986). Nucleotide sequence of the hag gene encoding flagellin of Escherichia coli. Journal of Bacteriology 168, 14791483.

LAEMMLI, U. K. (1970). Cleavage of structural proteins during the assembly of the head of bacteriophage T4. Nature, London 227, 680-685.

LaM, J. S., LaM, M. Y. C., MacDonald, L. A. \& HANCOCK, R. E. W. $(1987 a)$. Visualisation of Pseudomonas aeruginosa $\mathrm{O}$-antigens by using a protein A-dextran-colloidal gold conjugate with both immunoglobulin $G$ and immunoglobulin $M$

monoclonal antibodies. Journal of Bacteriology 169, 3531-3538.

Lam, J. S., MacDonald, L. A., Lam, M. Y. C., Duchesne, L. G. M. \& Southam, G. (1987b). Production and characterisation of monoclonal antibodies against serotype strains of Pseudomonas aeruginosa. Infection and Immunity 55, 1051-1057.

LAWN, A. M. (1977). Comparison of the flagellins from different flagellar morphotypes of Escherichia coli. Journal of General Microbiology 101, 121-130.

LAWN, A. M., ØRSKov, I. \& ØRSKov, F. (1977). Morphological distinction between different $\mathrm{H}$ serotypes of Escherichia coli. Journal of General Microbiology 101, 111-119.

MACNAB, R. M. (1987). Flagella. In Escherichia coli and Salmonella typhimurium. Cellular and Molecular Biology, pp. 70-83. Edited by F. C. Neidhardt, J. L. Ingraham, K. B. Low, B. Magasanik, M. Schaecter \& H. E. Umbarger. Washington, DC: American Society for Microbiology.

ØRSKOV, F. \& ØRSKOV, I. (1984). Serotyping of Escherichia coli. Methods in Microbiology 14, 43-112.

Towbin, M., Staehelin, T. \& GoRdon, J. (1979). Electrophoretic transfer of proteins from polyacrylamide gels to nitrocellulose sheets: procedure and some applications. Proceedings of the National Academy of Sciences of the United States of America 76, $4350-4354$.

Voller, A., Bidwell, D. E. \& Bartlett, A. (1976). Enzyme immunoassays in diagnostic medicine. Bulletins of the World Health Organisation 53, 55-65. 